
\title{
$\begin{array}{ll}\text { Research Square } & \begin{array}{l}\text { Preprints are preliminary reports that have not undergone peer review. } \\ \text { They should not be considered conclusive, used to inform clinical practice, } \\ \text { or referenced by the media as validated information. }\end{array}\end{array}$
}

\section{Clinical and Imaging Features of The Ovarian Cystadenofibroma or Adenofibroma and Their Correlation With Pathological Findings}

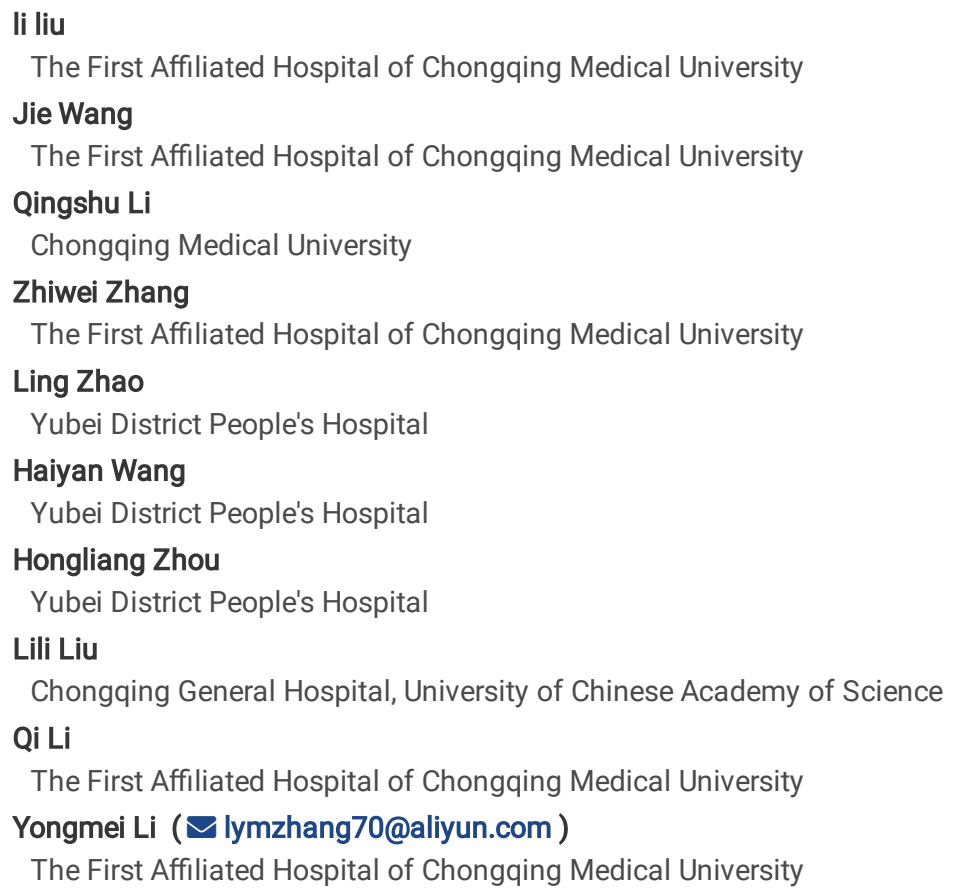

\section{Research Article}

Keywords: Ovarian Cystadenofibroma, Adenofibroma, Laboratory Results, Pathological Findings, Computed Tomography, Magnetic Resonance Imaging

Posted Date: September 1st, 2021

DOl: https://doi.org/10.21203/rs.3.rs-842206/v1

License: () (i) This work is licensed under a Creative Commons Attribution 4.0 International License. Read Full License 


\section{Abstract \\ Purpose}

To improve the accuracy of the preoperative diagnosis of ovarian cystadenofibroma (CAF) or adenofibroma (AF).

\section{Methods}

Clinical symptoms, laboratory results, imaging features, and pathological results of 11 patients with 11 histologically proven ovarian CAF/AF were reviewed retrospectively. The computed tomography (CT) and magnetic resonance imaging (MRI) features of the tumor including location, number, size, internal characteristics, CT density or MRI signal intensity, enhancement performance, lymphadenopathy, and amount of ascites were comprehensively evaluated.

\section{Results}

With regard to the clinical findings, results of serum cancer antigen 125 (CA125), cancer antigen 199 (CA199), and other laboratory tests were normal in most cases $(81.8 \%, 9 / 11)$. The imaging features of the 11 lesions were as follows: unilateral occurrence, well-defined boundary, round or roundish, unilocular $(63.6 \%, 7 / 11)$, cystic mass $(81.8 \%, 9 / 11)$, multilocular $(27.3 \%, 3 / 11)$, black sponge sign $(18.2 \%, 2 / 11)$, carpet sign $(81.8 \%, 9 / 11)$, residual ovary sign $(45.5 \%, 5 / 11)$, papillary nodule clusters $(45.5 \%, 5 / 11)$, and small vesicle with an acute angle to the inner cyst wall $(18.2 \%, 2 / 11)$. The signal intensity of the solid component was isointense or hypointense on T2-weighted imaging and hypo intense on diffusion-weighted imaging. The CT value of the lesions ranged from 21 Hounsfield units $(\mathrm{Hu})$ to $45 \mathrm{Hu}$. A slight to moderate enhancement degree occurred in more than half of the lesions $(54.5 \%, 6 / 11)$. No or a small amount of ascites was present in these cases. Histologic examination revealed SCAF $(45.5 \%, 5 / 11), \operatorname{SAF}(27.3 \%, 3 / 11)$, and borderline CAF/AF $(27.3 \%, 3 / 11)$. No necrosis, hemorrhage, or calcification was observed in any of these masses.

\section{Conclusion}

Ovarian CAF/AF usually mimics malignancy, demonstrating a cystic mass combined with solid component on CT or MR images. The integrity of case data including laboratory results and imaging features can help radiologists make an accurate preoperative diagnosis. No or a small amount of ascites may narrow the differential diagnosis.

\section{Introduction}

Ovarian cystadenofibroma (CAF) is a relatively uncommon tumor with an incidence of 1.7\%, as reported by Bohyun et al [1]. In 1974, Czernobilsky et al [2] described CAF as a benign tumor, creating a histologically distinct category from benign cystadenoma. Both CAF and adenofibroma (AF) of the ovary are considered to be deprived from germinal epithelium depression [3]. AF is composed of predominate benign stroma and neoplastic glandular. When a large cystic space presents in glandular elements [4], or when there is more than one cyst $>1 \mathrm{~cm}$ within the dense fibrous stroma [2, 5], the tumor is deemed as CAF. Although it is reported that most patients who develop ovarian CAF/AF are postmenopausal [4], there are still opportunities for woman of childbearing age to develop the disease, and most patients developing CAF/AF also have no characteristic symptoms.

The most frequently presenting symptom of both CAF/AF and malignant tumor of the ovary may be a palpable abdominal or pelvic mass or a cystic mass combined with solid component on computed tomography (CT) or magnetic resonance images (MRI). However, these require totally different treatment strategies, management, and prognoses. In addition, CAF/AF can be classified as benign or borderline based on the proliferation of epithelial cells. Benign CAF/AF may be treated by simple cystectomy or oophorectomy, whereas borderline tumors require hysterectomy, bilateral oophorectomy, and possibly omentectomy. Therefore, before surgery, benign CAF/AF must be accurately differentiated from borderline tumors of the ovary. However, differential diagnosis using tissue biopsy of the deep pelvic cavity is too harmful to patients of childbearing age with benign ovarian tumors. Consequently, in both entities, noninvasive imaging methods play important roles in accurate differential diagnosis, individualized treatments, and evaluation of therapeutic effects.

Previous studies [6, 7] have used MRI to analyze the components of CAF/AF, because of its greater soft-tissue contrast resolution. These studies pointed out the low T2-weighted signal intensity and enhancement of the solid component in CAF/AF. To the best of our knowledge, previous studies [1-7] used CT or MRI findings of CAF/AF including black sponge sign. However, not all CAF/AF contain typical features, as some are usually similar to malignant or other benign tumors, such as fibrothecomas, Brenner tumors, and so forth.

In this study, we attempt to comprehensively analyze the clinical and imaging characteristics of ovarian CAF or AF and their correlation with pathological findings, with the aim of improving the accuracy of preoperative diagnosis of this entity.

\section{Patients And Methods}


The study protocol was approved by ethics review committees of the First Affiliated Hospital of Chongqing Medical University in accordance with the laws and customs of China. Written informed consent was obtained from all patients before CT and MRI scans.

\section{Patient Population}

We retrospectively collected and analyzed the clinical data, imaging characteristics, and pathologic data of 11 patients with postoperative histopathologically proven ovarian CAF/AF who were treated at the First Affiliated Hospital of Chongqing Medical University from May 2013 to October 2020. The mean age of all patients was 43.5 years (range, $28-83$ years). All patients underwent surgery within 2 weeks after completing CT or MRI scans.

\section{CT and MRI Protocols}

Three patients underwent CT scan exclusively with a 128 multislice-spiral CT (GE Discovery CT750 HD system). The scanning range was from the top of the diaphragm to the inferior pubic symphysis, and the scanning protocol was as follows: slice thickness, 5 mm; interaction gap, 5 mm; pitch, 1.375 ; matrix, $512 \times 512$; tube voltage, $120 \mathrm{kV}$; and tube current, 200-500 mA. All three cases underwent contrast-enhanced CT scans with intravenous bolus injection of contrast medium (Lohexol, Omnipaque; GE Healthcare, Shanghai, China) at a rate of $3 \mathrm{~mL} / \mathrm{s}$ and a dose of $450 \mathrm{mgl} / \mathrm{kg}$ body weight. Images were acquired at 20-30 seconds, 60-90 seconds, and 120-150 seconds after the administration of contrast medium. Multiplanar reformation, maximum intensity projection, and volume rendering were conducted on the post processing workstation.

Seven patients underwent MRI examination exclusively with a 3.0 Tesla whole-body MRI system (Signa HDxt, GE Medical Systems, Milwaukee, Wisconsin) using a body phased-array coil. The transverse planes covered the entire area of the abdomen (ranging from top of the diaphragm to the inferior pubic symphysis). The following sequences were obtained: axial fast spoiled gradient recalled echo T1-weighted imaging (FSPGR-T1WI; FA/time of repetition [TR]/time of echo [TE], 80\%/200 ms/1.8 ms), slice thickness, $5 \mathrm{~mm}$; gap, $1 \mathrm{~mm}$; matrix $320 \cdot 224$; field of view, $40 \mathrm{~cm}$; 1 excitation; axial T2-weighted imaging (T2WI) using fast recovery fast spin echo (FRFSE; TR/TE, $4220 \mathrm{~ms} / 90.2 \mathrm{~ms}$ ); axial fast spin echo (FSE) T2WI with fat saturation (TR/TE, $4260 \mathrm{~ms} / 108.6 \mathrm{~ms}$ ); sagittal FSE T2WI (TR/TE, $3660 \mathrm{~ms} / 108 \mathrm{~ms}$ ). Axial diffusion-weighted imaging (DWI) was performed with a b value of $800 \mathrm{~s} / \mathrm{mm}^{2}$ using spin echo echo-planar imaging (SE-EPI; TR/TE, $5500 \mathrm{~ms} / 63.9 \mathrm{~ms}$ ). Apparent diffusion coefficient (ADC) maps were generated automatically.

We performed contrast-enhanced T1WI liver acquisition with volume acceleration 3D with fat saturation (TR/TE, $4 \mathrm{~ms} / 2 \mathrm{~ms}$ ) in the axial immediately after the injection of gadopentetate dimeglumine $(0.1 \mathrm{mmol} / \mathrm{kg}$ body weight, Magnevist; Bayer Schering) injected at a rate of $2-3 \mathrm{~mL} / \mathrm{s}$ and then repeated at $30,60,90$, and 120 seconds into the examination. The scanning parameters were as follows: slice thickness, 4 mm; gap 2 mm; matrix, 320 . 224; field of view, $40 \mathrm{~cm}$; and 0.7 excitation. Fat-suppressed spoiled gradient-echo T1WI was then performed in the sagittal or coronal planes.

In addition, one patient underwent both CT and MRI examination, the scanning protocol of which was as mentioned above.

\section{Observed Indicator}

Clinical data. Each patient's medical records contained the following: age, clinical symptoms, laboratory results, and complications.

Pathological data. All existing specimens were independently reviewed by two pathologists (Q.S.L. with 25 years of experience with pathologic diagnosis of the ovary; H.L.Z. with 6 years of experience with pathologic diagnosis). The pathological features of gross and histopathologic sections were recorded, respectively.

Image analysis. CT and MRI images were reviewed independently by two radiologists blinded to the pathological results (L.L. and Y.M.L.) who had 10 and 26 years of experience in gynecologic imaging, respectively. Any discrepancies were resolved by consensus. The following CT and MRI features were recorded for each case: size of the mass (maximum diameter), location (left or right side), shape (round, roundish, or lobulated), boundary (clear or obscure), interior structure patterns, maximum thickness of the cyst wall/septa, pre-contrast CT value or MR signal intensity, enhancement degree, and amount of ascites. According to the radiological evidence of the solid/cystic portion, the mass interior structure was classified into five patterns: (i) pattern I, pure cystic (no radiological evidence of solid; with or without septa); (ii) pattern II, cystic with papillary nodules or thickened cyst wall (solid component accounted for less than one-third; with or without septa); (iii) pattern III, mixed solid and cystic component (solid component accounted from one-third to two-thirds; with or without septa); (iv) pattern IV, solid with cysts or necrosis (solid component accounted for more than two-thirds); (v) pattern V, completely solid (no radiological evidence of cysts or necrosis). Patterns I and II were defined as cystic, pattern III as cystic-solid, and patterns IV and V as solid. The MR signal intensity of the masses was rated as hypointense (lower signal than myometrium), isointense (similar signal to myometrium), or hyperintense (higher signal than myometrium). The enhancement degree on CT and MR images were rated as slight (weaker than muscles), moderate (between muscles and myometrium), and intensive (more obvious than myometrium). The amount of ascites was divided into three degrees: (i) degree I, small (confined to Douglas' cul-de-sac); (ii) degree II, moderate (above the level of the uterine fundus); and (iii) degree III, large (abdominal distention caused by ascites). If the short axis of the lymph node was longer than $6 \mathrm{~mm}$ in the pelvic, retroperitoneal, or mesenteric region, lymphadenopathy was considered positive.

\section{Results}




\section{Clinical Characteristics and Laboratory Results}

In our study, patients with CAF/AF developed a palpable abdominal/pelvic mass (81.8\%, 9/11), abdominal distention (18.2\%, 2/11), abdominal pain $(18.2 \%, 2 / 11)$, or a small amount of vaginal bleeding $(18.2 \%, 2 / 11)$. Among the 11 patients, both serum cancer antigen 125 (CA 125 ; normal reference, $\leq 35 \mathrm{U} / \mathrm{mL}$ ) and cancer antigen 199 (CA199; normal reference, $\leq 27 \mathrm{U} / \mathrm{mL}$ ) were elevated in two patients (cases 9 and 10). No patients in this study had elevated serum human epididymis protein 4 (HE 4), carcinoembryonic antigen (CEA), or alpha-fetoprotein (AFP) levels. Laparoscopy was performed in 10 patients, and laparotomy was performed in the remaining 1 patient. Ovary solid mass was detected in 2 patients, cystic-solid mass was found in 1 patient during operations, and cystic masses of the ovaries was noted in the remaining 8 patients. Table 1 lists the clinical data.

A total of 7 cases were diagnosed as AF, but 4 were CAF, and complications were observed during operations in 6 cases. Infarction was observed in 1 case, torsion $\left(720^{\circ}\right)$ and endometriosis in 1 case, uterine fibroid in 1 case, dilatation tubal and uterine fibroid in 1 case, endometriosis in 1 case, and serous cystadenoma in one case. In the specimens dissected during the operations, we observed papillary nodular structures inside the cyst wall in cases 1, 2, 3, and 8 (Fig. 1).

\section{CT and MRI Characteristics}

Eleven tumors occurred in 11 cases unilaterally, including 5 cases in the left ovary and 7 cases in the right. All tumors demonstrated a well-defined boundary and were round or analogous round in shape. The maximum diameter of these tumors ranged from 6-21 cm. One cystic-solid mass, $1 \mathrm{solid}$ mass, and 9 cystic masses were found in this study. The features of the tumors, including maximum diameter, maximum thickness of the cyst wall/septa, interior structure pattern, tumor attenuation on CT image, signal intensity on MRI, and enhancement degree, were demonstrated clearly and are described in Table 2.

The interior structure of the masses in this study was classified into five patterns based on the radiological evidence of the solid/cystic portion. Pattern I, pure cystic, seen in 1 case $(9 \%, 1 / 11)$, with no radiological evidence of solid shown. Pattern II, cystic with papillary nodules or thickened cyst wall, seen in 8 cases $(73 \%, 8 / 11)$; the solid component accounted for less than one-third. Pattern III, mixed solid and cystic component, seen in 1 case ( $9 \%$, $1 / 11)$; the solid accounted from one-third to two-thirds. Pattern V, completely solid mass, seen in 1 case $(9 \%, 1 / 11)$; no cysts or necrosis were shown on CT or MRI. There were no cases of pattern IV in this study. Among the cystic/cystic-solid masses, 3 cases $(27.3 \%, 3 / 11)$ were multilocular, whereas 7 cases $(63.6 \%, 7 / 11)$ were unilocular (Table 2$)$. The solid component in our study manifested as papillary nodule clusters inside the cyst wall or as thickened cyst wall/septa, the characteristic signal intensity of which was mainly isointense or hypointense on T2WI (Fig. 2), hypointense on DWI, and the mean $\mathrm{ADC}$ value was $(1.39 \pm 0.25) \cdot 10^{-3} \mathrm{~mm}^{2} / \mathrm{s}$. The CT value of the solid region ranged from $21 \mathrm{Hu}$ to $45 \mathrm{Hu}$. On contrast CT or MRI scans, the degree of solid component was none $(18.2 \%, 2 / 11)$, slight $(36.4 \%, 4 / 11)$, or moderate $(18.2 \%, 2 / 11)$ (Fig. 2), respectively. However, in cases 1 , 3 , and 10 , the signal intensity of the solid component demonstrated T2-isointense, a little higher on DWI (Fig. 3), intensive on contrast-enhanced MRI (Fig. 3), and the mean $A D C$ value was $(1.28 \pm 0.15) \cdot 10^{-3} \mathrm{~mm}^{2} / \mathrm{s}$.

In addition, we observed some specific MRI signs in some lesions. On T2WI, very high signal intense tiny cysts were presented in very low signal intense solid components in cases 1 and 4 (18.2\%, 2/11); small vesicles were seen inside the wall of the cysts in cases 2 and 8 (18.2\%, $2 / 11)$, which were with an acute angle to the inner cyst wall (Fig. 2). Localized thickening of the cyst wall/septa was observed in cases 1, 2, 3, 4, 6, 7, 8, 10, and 11 (81.8\%, 9/11; Fig. 4); a trabecular solid area between smoothly thickened septa was shown in cases 1 and 10 (18.2\%, 2/11); residual ovary follicles and stromal signals were seen in cases $2,3,4,5$, and $8(45.5 \%, 5 / 11)$ on MRl; and clusters of papillary nodules were demonstrated in cases $1,2,3,6$, and 8 $(45.5 \%, 5 / 11$; Fig. 4). Ascites was present in cases 1, 3, 4, and 7 (36.4\%, 4/11), the amount of which was small, confined to Douglas's-de-sac. No cases exhibited a positive lymphadenopathy.

\section{Pathological Basis}

A definitive histopathological diagnosis was obtained in all 11 cases. Pathologic findings confirmed that these masses were SCAF in 5 (45.5\%) of the 11 patients, SAF in 3 patients (27.3\%), borderline CAF in 2 patients (18.2\%; one patient was borderline SCAF, the other was borderline ECAF), and borderline AF in 1 patient (9.1\%). Immunohistochemical staining was performed on case 7, which stained positive for PR, vimentin, CD56, CD31 and partly positive for ER, Ki-67, WT-1, SMA, CD99, inhibin-a, respectively. No necrosis, hemorrhage, or calcification was observed in any of these masses.

We correlated imaging features with the pathologic findings after surgery. Dense fibrous stromal proliferation was responsible for the hypointensity seen on T2WI (Fig. 1). A diffusely or partially thickened wall or clusters of papillary nodules at CT or MR was also found upon pathologic examination to be related to proliferating dense fibrous stromal. In addition, small cystic locules were shown in the T2 dark signal intensity solid component in 2 cases, indicating multiple small saclike glandular structures within dense fibrous tissue at pathologic examination (Fig. 1).

Upon hematoxylin-eosin staining, photomicrographs at ·40 magnification showed that the epithelium was columnar or cubic, which was serious in 10 cases and endometrioid in the remaining 1 case. In 3 cases, epithelial cells were stratified with obvious cell atypia, and the above morphological range exceeded $10 \%$, resulting in a diagnosis of borderline tumors. In addition, the epithelium covering the small saclike glandular structures was the same as that of the cyst wall. High-power photomicrograph at $\times 100$ showed papillary nodular structures inside the cyst wall (Fig. 1).

\section{Discussion}


Most cases of ovarian CAF/AF demonstrate a cystic mass combined with a solid component, which makes accurate preoperative diagnosis difficult. Misdiagnosis may increase the psychological burden of patients as well as the occurrence of unnecessary interventions. Although pathological diagnosis is the gold standard, image diagnosis is superior in comprehensively determining the benign, malignant, adjacent condition, and the treatment choice before surgery [1]. In this study, we investigated the imaging characteristics of 11 patients with ovarian CAF/AF to correlate their respective imaging characteristics with histopathologic findings. Imaging features of these tumors included a cluster of papillary nodular structures inside the cyst wall $(45.5 \%, 5 / 11)$, diffusely or partially thickened wall/septa $(81.8 \%, 9 / 11)$, unilocular $(63.6 \%, 7 / 11)$, and a well-encapsulated, cystic mass, and in some cases black sponge sign (18.2\%, 2/11). More than half of the cases had pattern II (72.7\%, 8/11), papillary nodule clusters or thickened wall/septa (solid accounted for less than one-third) contained in the cystic masses.

In the current study, $63.6 \%(7 / 11)$ of the lesions were unilocular. However, Dae et al [4] reported a unilocular rate of $7.7 \%$. We speculated that this difference could be ascribed to the small samples of both studies. The morphology of the solid component demonstrated a mass (18.2\%, $2 / 11)$, a solid region with a septum $(18.2 \%, 2 / 11)$, a diffusely or partially thickened wall $(81.8 \%, 9 / 11)$ like carpet, or a cluster of papillary nodular structures inside the cyst wall $(45.5 \%, 5 / 11)$. Yen et al [6] referred to the diffusely thickened wall-like carpet as the "carpet sign." The rate of papillary nodular clusters in our study was consistent with that of previous studies (range, 33-80\%) [8-12]. Our study demonstrated that a diffusely or partially thickened wall was the most common feature $(81.8 \%, 9 / 11)$, and rate was close to that reported by Bohyun [1]. Consistent with previous studies [4-8, 11], the solid component demonstrated an isointense or hypointense signal on T2WI, and a hypointense signal intensity on DWI, similar to skeleton muscles. A T2 dark signal intensity solid component containing small cystic locules on MRI was called the black sponge sign, which was observed in only 2 cases (18.2\%, $2 / 11$ ) in our study, a rate that is lower than that reported in previously studies $[2,4,6-8]$.

In addition, case 5 in our study combined with infarction presented a solid mass, the signal intensity of which was also distinctive in this group but consistent with the other tumors accompanied by infarction: isointense on T2WI, hypointense on DWI, and lack of enhancement.

Ovarian CAF/AF is an epithelial-derived tumor [1-3]. It is categorized into 2 subtypes based on the cytologic features of the glandular element: benign and borderline. In our study, 8 benign and 3 borderline cases were included. The solid mass, diffusely or partially thickened wall/septa and papillary nodular clusters inside the cyst wall represented a dense fibrous component, correlated with histopathologic findings. Microscopically, small cystic glandular structures scattered in the fibrous stroma manifested very high signal intense tiny cysts presented in very low signal intense solid components on T2WI, which was called the black sponge sign. Moreover, we observed 1 cystic-solid mass, 2 solid masses, and 8 cystic masses during surgical operations, which were not consistent with CT/MRI (1 cystic-solid mass, 1 solid mass, and 9 cystic masses). The main discrepancy was case 4 combined with torsion of pedicle. The inside wall of the cyst in cases 6 and 10 was described as smooth during the surgical operations, but papillary nodular clusters of structures were shown inside the wall on T2WI. The above imaging findings were confirmed by pathology.

$\mathrm{CAF} / \mathrm{AF}$ is a rare ovarian tumor. A previous study reported that the onset age span is wide (range, 23-70 years) and most patients who developed the disease are postmenopausal [4]. Abdominal/pelvic mass, abdominal distension or pain, and postmenopausal vaginal bleeding are the main symptoms. In the present study, postmenopausal women accounted for $72.7 \%(8 / 11)$, and a total of 8 patients $(72.7 \%, 8 / 11)$ manifested abdominal/pelvic mass. This was consistent with past reports. However, in this study, only 2 patients $(18.2 \%, 2 / 11)$ developed postmenopausal vaginal bleeding. In addition, the levels of both CA125 and CA199 were elevated in only 2 cases (18.2\%, 2/11). One case was accompanied by endometrial implantation, and the other was accompanied by cervical polyp. Those complications may account for the elevation we presumed. No patient enrolled in our study had elevated serum HE 4, CEA, or AFP levels.

In addition, we observed several novel findings in our study. First, a small vesicle with an acute angle to the inner wall of the cysts was seen in cases 2 and 8 . Second, a solid region with a septum, such as honeycomb (case 1) and onion peel (case 10). Third, among all 11 cases in our study, cases 2 , 3 , 4,5 , and 8 demonstrated residual ovary follicles and stromal signals on MRI, whereas these were relatively rare in borderline cases, seen only in case 3 . We referred to this as residual ovary sign. It is well-known that MRI has the highest resolution for the ovary, but cases 6 and 9 (benign) underwent CT only. That is probably why the ovaries in the 2 benign cases did not show as clear, like we presumed. Despite the selection of examination methods (CT or MRI), we speculated that it may also be related to the natural quality of the begin, borderline, or malignant tumor. Fourth, ascites was not found in pure benign CAF/AF in our study. Only 2 borderline and 2 benign lesions in the current study were combined with ascites, the latter of which were case 4 complicated with a torsion and case 7 complicated with dilatation of the fallopian tube, local congestion, and bleeding in the wall with scattered chronic inflammatory cell infiltration. We speculated that the complications above may be accounted for by the production of ascites. In conclusion, the amount of ascites associated with $\mathrm{CAF} / \mathrm{AF}$ is small, which is significantly different from ovarian malignant tumors, such as serous cystadenocarcinoma.

Our study has some limitations. First, to verify the novel findings found in the current study, a prospective study with a large sample containing all classifications of CAF/AF is needed. Second, because the surgeon may not be the same gynecologist in all cases, the consistency between surgical and image findings remains to be further verified.

\section{Conclusion}

Ovarian CAF/AF usually mimics malignancy, appearing as a cystic mass combined with a solid component on CT and MRI. Therefore, the integrity of case data, including laboratory results and imaging features, is particularly important. The characteristics of the solid region are T2-hypointense, DWhypointense, and slight or moderately enhanced. Black sponge sign, residual ovary sign, and carpet sign are often seen on MRI in benign CAF/AF. If the

Page 5/11 
solid component signal intensity shows slightly higher on DWI and intense enhancement following contrast administration, borderline CAF/AF is more likely. In addition, normal level of CA125, CA199, HE4, and no or a small amount of ascites presented may narrow the differential diagnosis.

\section{Abbreviations}

CAF

Cystadenofibroma; AF:Adenofibroma; CT:Computed tomography; MRI:Magnetic resonance imaging;

\section{Declarations}

\section{Acknowledgments}

Not applicable.

\section{Funding}

Not applicable.

\section{Availability of data and materials}

Please contact the first author for data requests.

\section{Ethics approval and consent to participate}

The study protocol was approved by ethics review committees of the First Affiliated Hospital of Chongqing Medical University in accordance with the laws and customs of China in the study (code: 2021-029). Written informed consents were obtained from all patients before CT and MRI scans.

\section{Consent for publication}

Informed consent for publication was obtained from all participants.

\section{Competing interests}

The authors declare that they have no competing interests.

\section{Authors' contributions}

Qi Li and Yongmei Li contributed equally to this work and shared the corresponding author.

LL conceived of the study, and participated in its design and drafted the manuscript. JW, LLL, QSL, LZ, HYW and HLZ participated in the data analysis/data acquisition. YML and QL guaranteed the integrity of entire study. ZWZ participated in the data acquisition. All authors read and approved the final manuscript.

\section{Author details}

1Department of Radiology, the First Affiliated Hospital of Chongqing Medical University, 1 Youyi Road, Chongqing 400016, China. 2 Department of Nuclear Medicine, the First Affiliated Hospital of Chongqing Medical University, 1 Youyi Road, Chongqing 400016, China. 3 Department of Pathology, Chongqing Medical University, 1 Yixueyuan Road, Chongqing 400016, China. 4 Department of Radiology, Yubei District People's Hospital, 23 ZhongyangGongyuanBei Road, Yubei District, Chongqing 401120, China. 5 Department of Pathology, Yubei District People's Hospital, 23 ZhongyangGongyuanBei Road, Yubei District, Chongqing 401120, China. 6 Department of Radiology, Chongqing General Hospital, University of Chinese Academy of Science, 118 Avenue of Star Road, Chongqing 400000, China.

\section{References}

1. Bohyun K, Kyoung-Sik Cho, Seung HK, et al. CT and MRI findings of cystadenofibromas of ovary. Eur Radiol. 2004;14:798-804.

2. Czernobilsky B, Borenstein R, Lancet M. Cystadenofibroma of the ovary. A clinicopathologic study of 34 cases and comparison with serous cystadenoma. Cancer. 1974;34:1971-81.

3. Seidman JD, Mehrotra A. Benign ovarian serous tumors: a re-evaluation and proposed reclassification of serous "cystadenomas" and "cystadenofibromas". Gynecol Oncol. 2005;96:395-401.

4. Jung DC, Kim SH, Kim SH. MR imaging findings of ovarian Cystadenofibroma and Cystadenocarcinofibroma: clues for the differential diagnosis. Korean J Radiol. 2006;7:199-204.

5. Lee DH. A case of mucinous cystadenofibroma of the ovary. Case Rep Obstet Gynecol. 2014;2014:1-4. 
6. Tang YZ, Liyanage S, Narayanan P, et al. The MRI features of histologically proven ovarian cystadenofibromas-an assessment of the morphological and enhancement patterns. Eur Radiol. 2013;23:48-56.

7. Takeuchi M, Matsuzaki K, Harada M. Ovarian adenofibromas and cystadenofibromas: magnetic resonance imaging findings including diffusionweighted imaging. Acta Radiol. 2013;54:231-6.

8. Outwater EK, Siegelman ES, Talerman A, Dunton C. Ovarian fibromas and cystadenofibromas: MRI features of the fibrous component. J Magn Reson Imaging. 1997;7:465-71.

9. Alcázar JL, Errasti T, Mínguez JA, et al. Sonographic features of ovarian cystadenofibromas: spectrum of findings. J Ultrasound Med. 2001;20:915-9.

10. Fatum M, Rojansky N, Shushan A. Papillary serous cystadenofibroma of the ovary: Is it really so rare? Int J Gynaecol Obstet. 2001;75:85-6.

11. Jeong YY, Outwater EK, Kang HK. Imaging evaluation of ovarian masses. Radiographics. 2000;20:1445-70.

12. Atri M, Nazarnia S, Bret PM, et al. Endovaginal sonographic appearance of benign ovarian masses. Radiographics. 1994;14:747-60.

\section{Tables}

Table 1. Clinical features, laboratory results, surgical findings and pathological classification of the 11 patients with CAF/AF

\begin{tabular}{|c|c|c|c|c|c|c|c|c|c|c|c|c|c|c|c|}
\hline \multirow[t]{2}{*}{ No. } & \multirow[t]{2}{*}{ Age } & \multicolumn{4}{|c|}{ Clinical symptoms } & \multirow{2}{*}{$\begin{array}{l}\text { CA125 } \\
(\mathrm{U} / \mathrm{ml})\end{array}$} & \multirow{2}{*}{$\begin{array}{l}\text { CA199 } \\
(\mathrm{U} / \mathrm{ml})\end{array}$} & \multirow{2}{*}{$\begin{array}{l}\mathrm{HE} \mathrm{4} \\
(\mathrm{pmol} / \mathrm{L})\end{array}$} & \multicolumn{5}{|c|}{ Surgical findings } & \multirow{2}{*}{$\begin{array}{l}\text { Pathological } \\
\text { Classification }\end{array}$} & \multirow[t]{2}{*}{ Complications } \\
\hline & & $\mathrm{PM} / \mathrm{AM}$ & AP & $A D$ & VB & & & & $\mathrm{R}$ & $\mathrm{L}$ & $\begin{array}{l}\mathrm{C}- \\
\mathrm{S} \\
\mathrm{M}\end{array}$ & SM & $\mathrm{CM}$ & & \\
\hline 1 & 53 & + & & & & 13 & 3.7 & 54 & + & & & & + & $\begin{array}{l}\text { Borderline } \\
\text { SAF }\end{array}$ & Multiple uterine fibroids \\
\hline 2 & 83 & + & & & + & 5.4 & 1.6 & 65 & + & & & & + & SCAF & None \\
\hline 3 & 39 & + & + & & & 22.8 & 2.6 & 46 & & + & & & + & $\begin{array}{l}\text { Borderline } \\
\text { SCAF }\end{array}$ & None \\
\hline 4 & 51 & + & & & & 10.9 & 1.9 & 42 & & + & & + & & SAF & Torsion of pedicle, $720^{\circ}$ \\
\hline 5 & 56 & + & & & & 34.6 & 3.4 & 34 & + & & & + & & SCAF & Infarction; endometriosis \\
\hline 6 & 70 & + & & & & 9.7 & 1.3 & 59 & & + & & & + & SAF & None \\
\hline 7 & 43 & & & & + & 18.4 & 7.1 & 32 & + & & & & + & SCAF & $\begin{array}{l}\text { Dilatation tubal; uterine } \\
\text { fibroid }\end{array}$ \\
\hline 8 & 60 & & + & + & & 25.5 & 13.8 & 57 & + & & & & + & SCAF & SC, left \\
\hline 9 & 28 & + & & & & 71.9 & 62.9 & 28 & + & & & & + & SAF & endometriosis, bilateral \\
\hline 10 & 67 & & & + & & 117 & 28.4 & 57 & + & & & & + & $\begin{array}{l}\text { Borderline } \\
\text { ECAF }\end{array}$ & None \\
\hline 11 & 61 & + & & & & 12.5 & 1.9 & 54 & & + & + & & & SCAF & None \\
\hline
\end{tabular}

PM, Pelvic mass; AM, Abdominal mass; AD, Abdominal distension; AP, Abdominal pain; VB, Vaginal bleeding; SCAF, serous cystadenofibroma; SAF, serous adenofibroma; SC, serous cystadenoma; ECAF, Endometrioid cystadenofibroma; C-S M, Cystic-solid mass; CM, Cystic mass; SM, solid mass

Table 2 CT/MRI features of the 11 patients with CAF/AF

Max.D, maximum diameter; Iso, isointense; Hypo, hypointense; Hyper, hyperintense; M, mass; PN, Papillary nodules; RTCW, Regional thickened capsule wall; ML, multilocular; UL, unilocular; S, slight; M, moderate; I, intensive; N, none; Hu, hounsfield unit

\section{Figures}




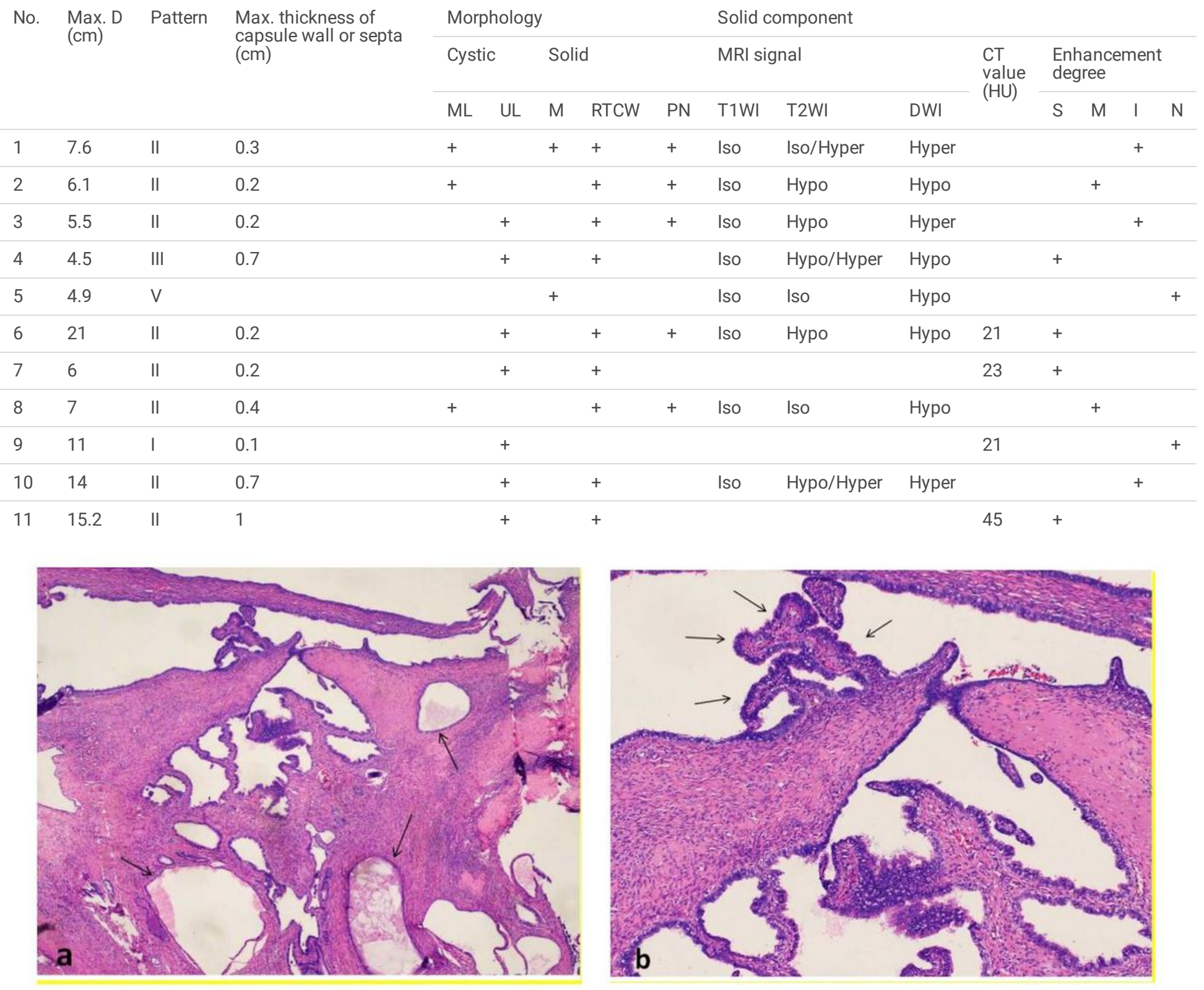

Figure 1

A 43-year-old woman with a serous papillary cystadenofibroma of the right ovary. a Low-power photomicrograph (original magnification, $\times 40$; hematoxylin - eosin (H-E) stain) shows multiple small saclike (arrows) glandular structures in dense fibrous stroma. b High-power photomicrograph (original magnification, $\times 100 ; \mathrm{H}-\mathrm{E}$ stain) shows papillary nodular structures inside the cyst wall (arrows). 

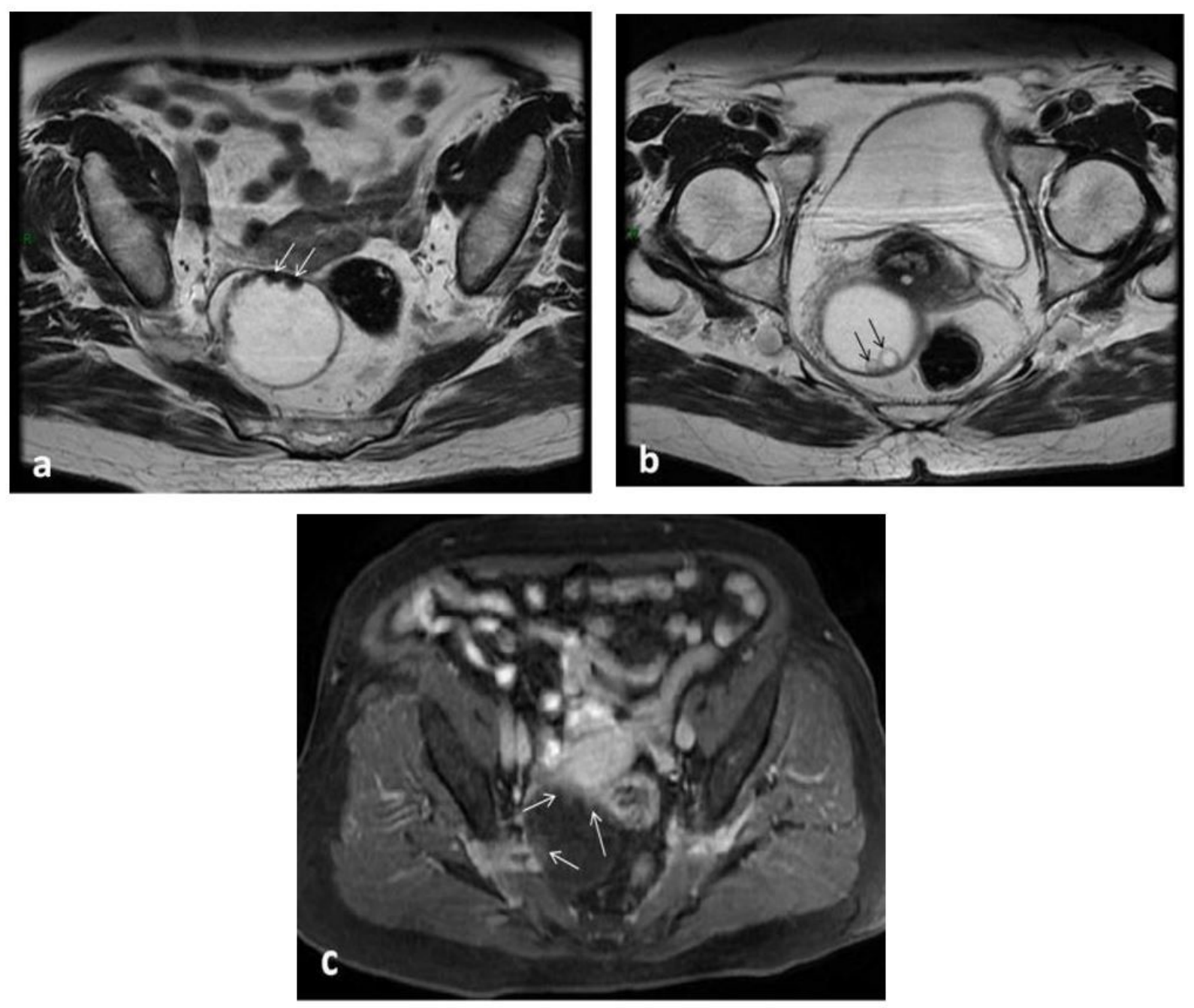

\section{Figure 2}

A 83-year-old woman with a serous cystadenofibroma. a Pre-contrast axial T2WI shows a unilocular cystic mass with the presence of focal nodular (arrows) inside the partly thickened wall of hypointense in the right ovary. b Pre-contrast axial T2WI shows small vesicle on the inner wall of the cysts with an acute angle to the wall (arrows). c Post-contrast axial T1WI LAVA 3D with fat saturation image shows mild enhancement of solid component (arrows). 

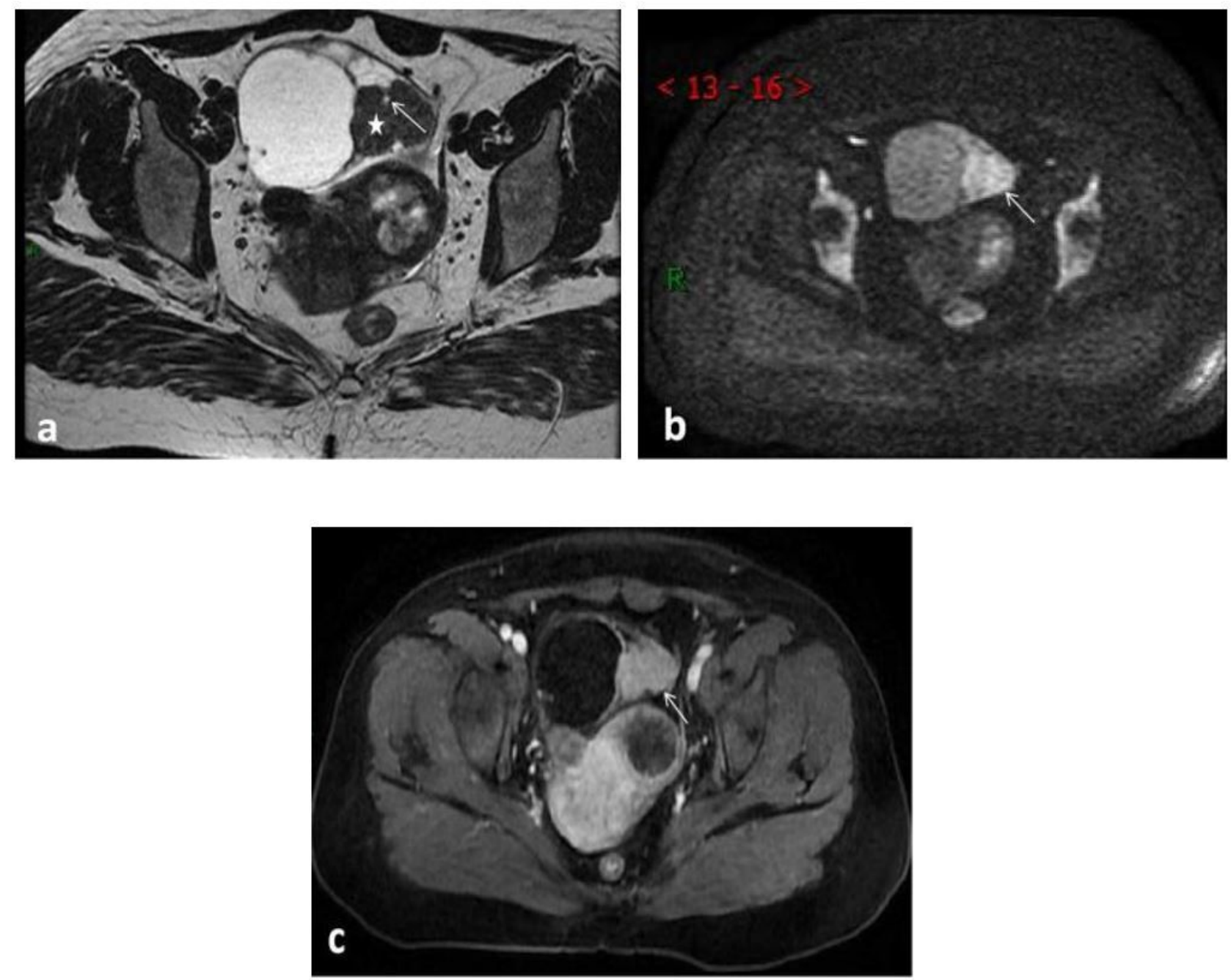

\section{Figure 3}

A 53-year-old woman with a borderline serous adenofibroma. a Pre-contrast axial T2WI shows a multilocular cystic-solid mass in the right ovary. The solid component (pentagram) demonstrates isointense with small hyperintense cystic locules (arrows). b Axial DWI shows the solid component with a little higher signal intensity (arrows). c Post-contrast axial T1WI LAVA 3D with fat saturation image shows intensive enhancement of solid component (arrows). 


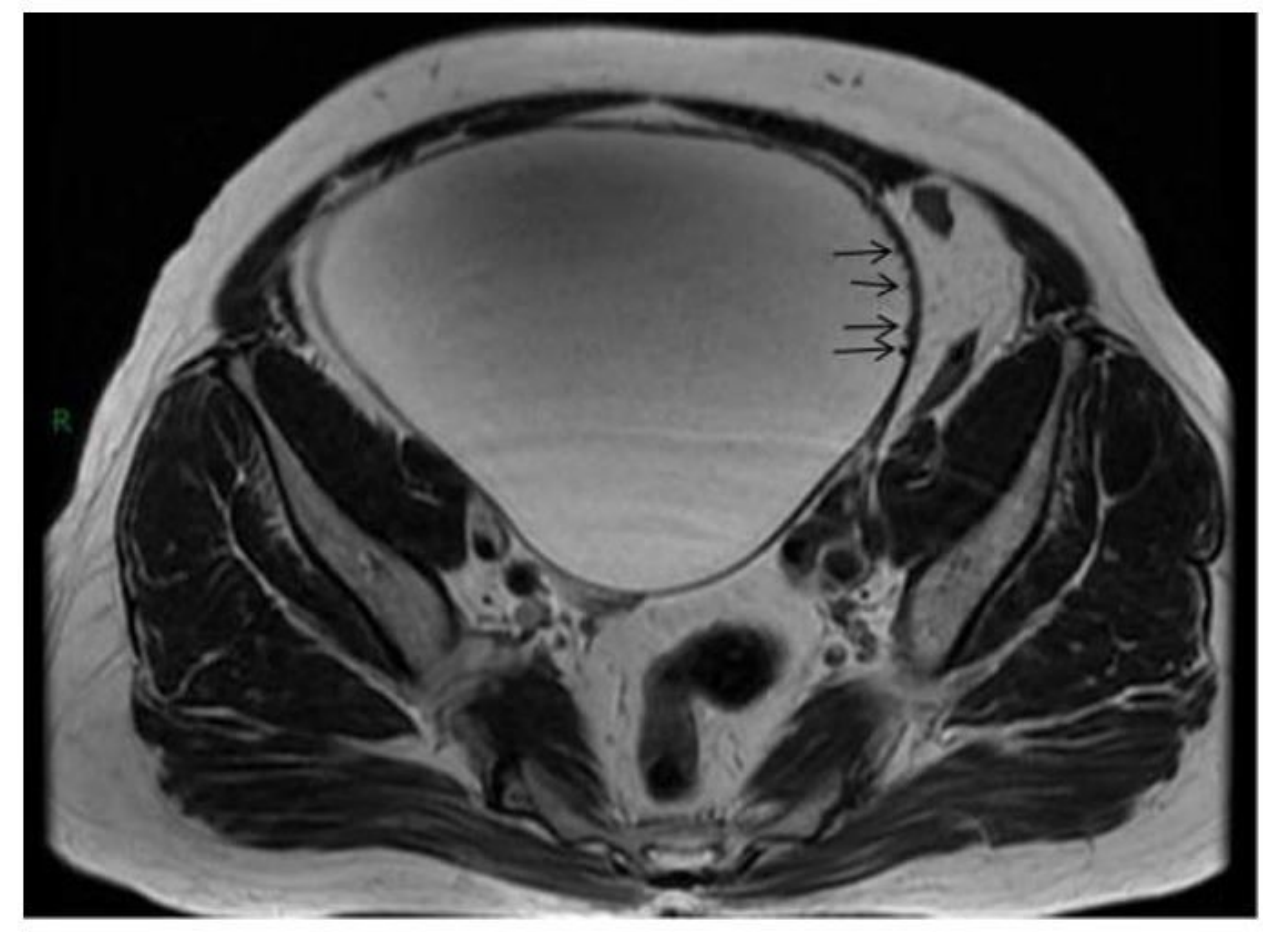

Figure 4

A 70-year-old woman with a borderline serous adenofibroma. Pre-contrast axial T2WI shows localized thickening of the cyst wall, and a cluster of papillary nodules inside the cyst wall (arrows). 